\title{
EXPERIMENTAL AND NUMERICAL ANALYSIS OF LIQUID COUNTERFLOW JETS: STUDY OF RESURGENT JET GENERATION
}

\author{
DIMITRI DOMINGIE ${ }^{1}$, JEAN-BAPTISTE DEUFF $^{2}$ \& OLIVIER PERELMAN ${ }^{2}$ \\ ${ }^{1}$ Airbus Safran Launchers, Les Mureaux, France. \\ ${ }^{2}$ DGA Hydrodynamics, France.
}

\begin{abstract}
The present study deals with plumes of water, called here resurgent flows, which can result from the impact of a liquid jet on a reverse-flow liquid surface. This phenomenon has to be taken into account in the conception of the industrial device. Most of the studies on this topic are oriented towards the air entrainment created by a plunging jet. The aim of this study is twofold: to characterize the main parameters about the phenomenology of resurgent jets and to predict the resurgence generation using a dual numerical and experimental approach. A large test campaign had been realized and assesses the influence of several parameters such as velocities, diameter of jets, impinging angle thanks to an injection pipe put on a moving platform. It appears that the phenomenon induces high levels of loads and cannot be neglected when singular conditions are met. Experimental results were faced to numerical results in order to validate models. These results show a great accordance between experiments and calculations and therefore the capabilities of the numerical code STAR-CCM+C) to restitute resurgent jets.

Keywords: counter-flow, free surface, impinging jet, resurgent jet, Volume-Of-Fluid.
\end{abstract}

\section{INTRODUCTION}

Plunging liquid flows are frequently encountered in nature as well as engineering applications. There are many examples of these phenomena such as mixing flows [1], breaking waves or jet-propelled navy vessels. Most research on that topic has focused on the initial plunging jet impact and the analysis of the resulting cavity shape evolution, pinch-off mechanics and the air entrainment [2]. Fontaine et al. [3] studied the post breaking phase (splashing) of waves with a Smoothed Particles Hydrodynamics (SPH) method. A liquid jet impinged a free surface at rest, both inclined. Deshpande et al. [4] proposed an experiment and numerical study with Volume Of Fluid (VOF) method to model a shallow inclination plunging jet in a free surface at rest. In Ref. [5], a vertical plunging jet is impinging a moving flow. Resurgent jets match to a deformation of the free surface, which results from an inclined water jet impinging a reverse-flow liquid surface. A better knowledge of the resurgent jet characteristic and its main driving parameter are necessary for a smarter design of structures subjected to these projected plumes of water. In this context, this study aims to provide the elements for an understanding of the resurgent jet phenomenon through a dual numerical and experimental approach.

The presentation of the work is as follows. First, a description of the experimental setup and measurements is given, followed by the analysis of the phenomenon observed during the test campaign. Then, a numerical approach is presented and the computational results are compared to experimental data. Finally, the relevance of the experimental setup and the numerical approach are discussed in the conclusions. 


\section{EXPERIMENTAL SETUP}

The tests have been performed in the B600 towing tank of DGA Hydrodynamics (France). This tank is $600 \mathrm{~m}$ long, $15 \mathrm{~m}$ wide and $7 \mathrm{~m}$ depth. The experimental set up module (Figs. 1 and 2) has been fixed on the carriage plat-form so that tests with counter velocity are performed. The water for the jet is pumped in a $1 \mathrm{~m}^{3}$ tank. The velocity of the jet is measured thanks to an ultrasonic flowmeter mounted on a long enough straight pipe. The experimental set up allowed three types of measurements with free or blocked jets:

- The free resurgent jet is characterized with high frame rate $(400 \mathrm{~Hz})$ cameras (in green on Figs. 2 and 3). The free surface is highlighted by a vertical laser sheet and its coordinates are obtained by images post processing. Thanks to cameras field of view calibration (Fig. 3), the water interface positions can be compared to CFD computations.

- In the blocked jet configuration, an instrumented shield (Fig. 4) with pressure transducers or force sensor is located in the resurgent jet zone (Figs. 1 and 2), as seen from the free resurgent jet configurations.

In order to avoid perturbations of the free surface during the transient phase (when the flow grows to reach the jet test conditions), we used a pipe system with an elbow, placed in front of the nozzle that deviates the jet out of the measurement area. When the right jet velocity is obtained, this system is quickly moved on the side, allowing the jet to impact the water surface.



Figure 1: Set up module in pressure measurement configuration. 


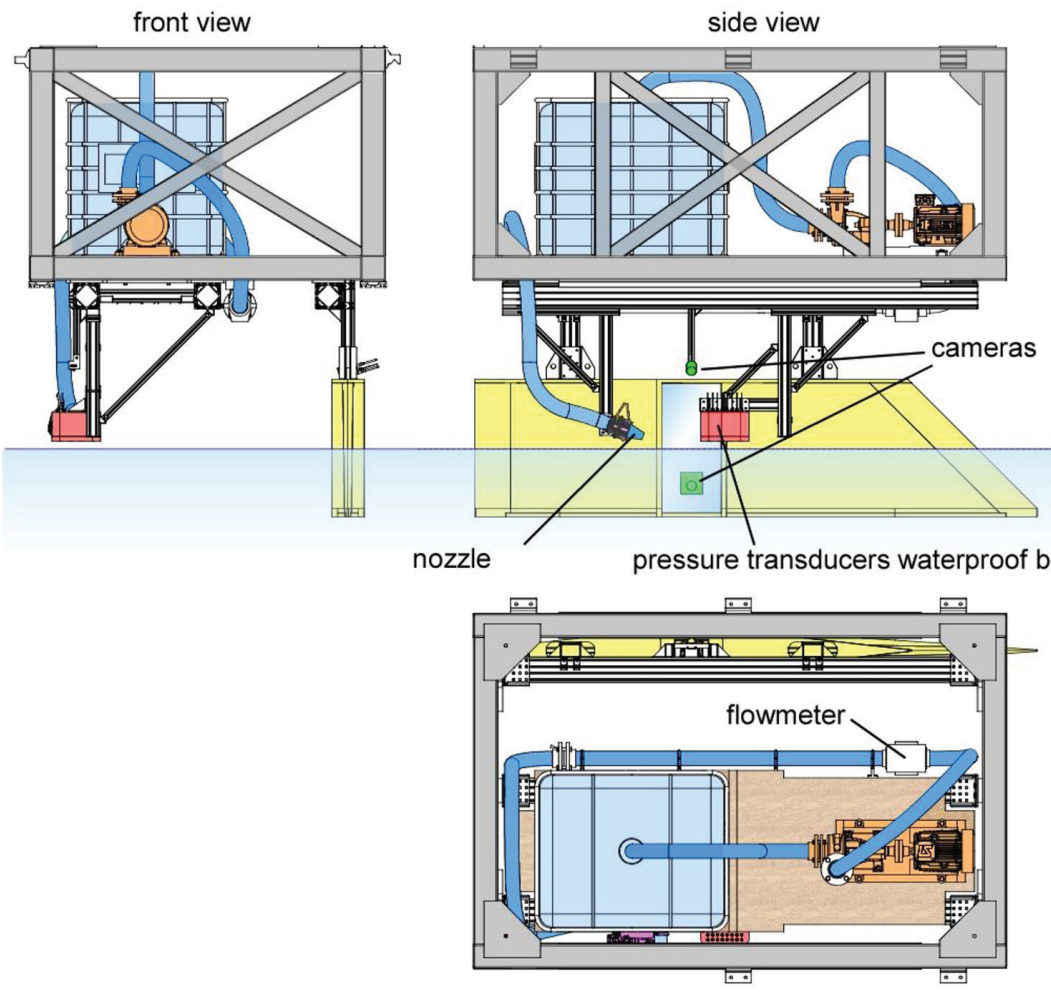

top view

Figure 2: Views of the set up in pressure measurement configuration.

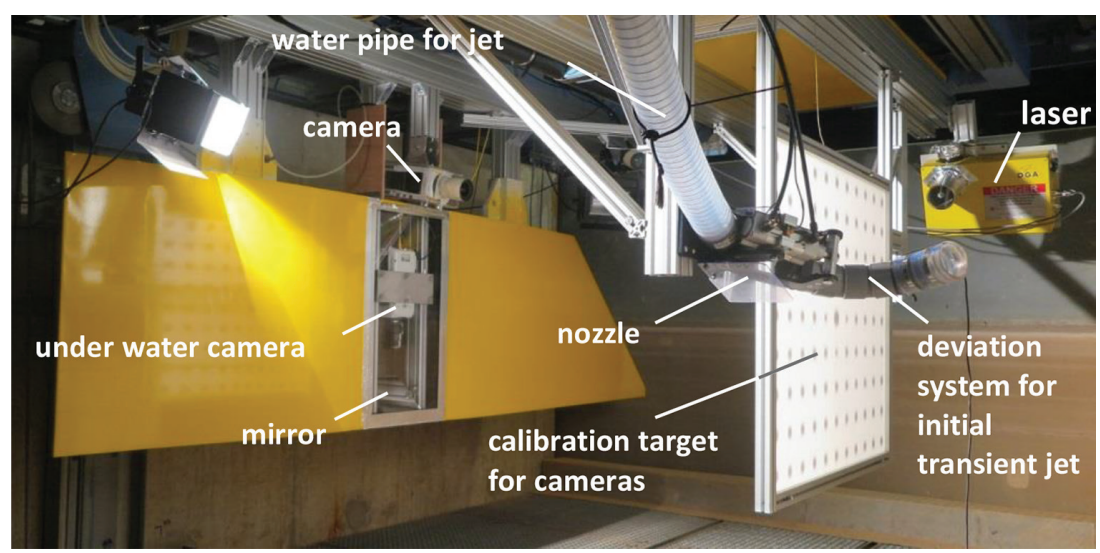

Figure 3: Set up in drydock during optical measurement system calibration. 

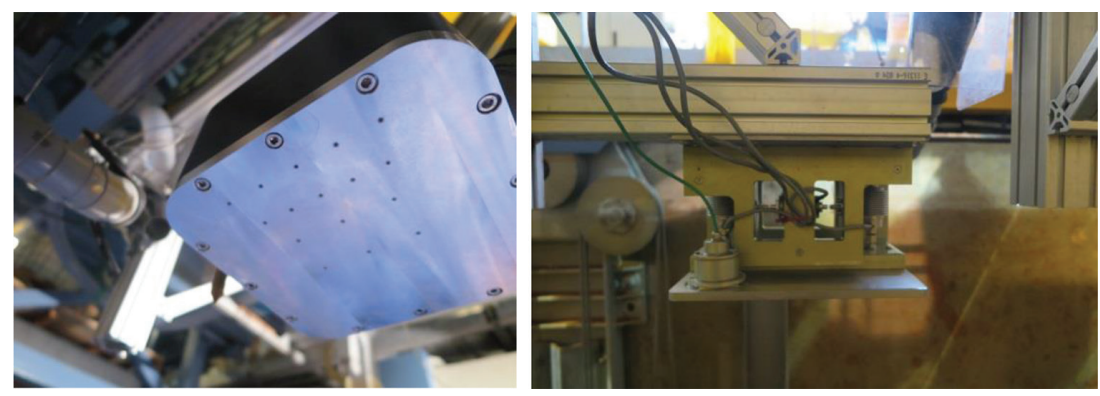

Figure 4: View of the plate with pressure transducers (left) and load measurement (right).

\section{PHENOMENOLOGY OF RESURGENT JETS}

This part aims to understand the phenomenology of resurgent jets. Both video and sensors highlight several resurgent jet life phases (see Fig. 5). In free jet conditions, the jet is impinging the free surface (1) generating circular plumes of water around the impact zone (the impinging jet penetrates deeper the free surface) (2). The resurgent jet grows and is fed by the counter-flow and moves towards the impinging jet, like wave breaking (3), until the interaction with the impinging jet. The stagnation point in the impact zone reaches the maximum depth (4). This interaction breaks both resurgent and impinging jets and the deformation of the free surface is drifted downstream (5). Finally, the impinging jet regains its integrity until a new cycle begins.
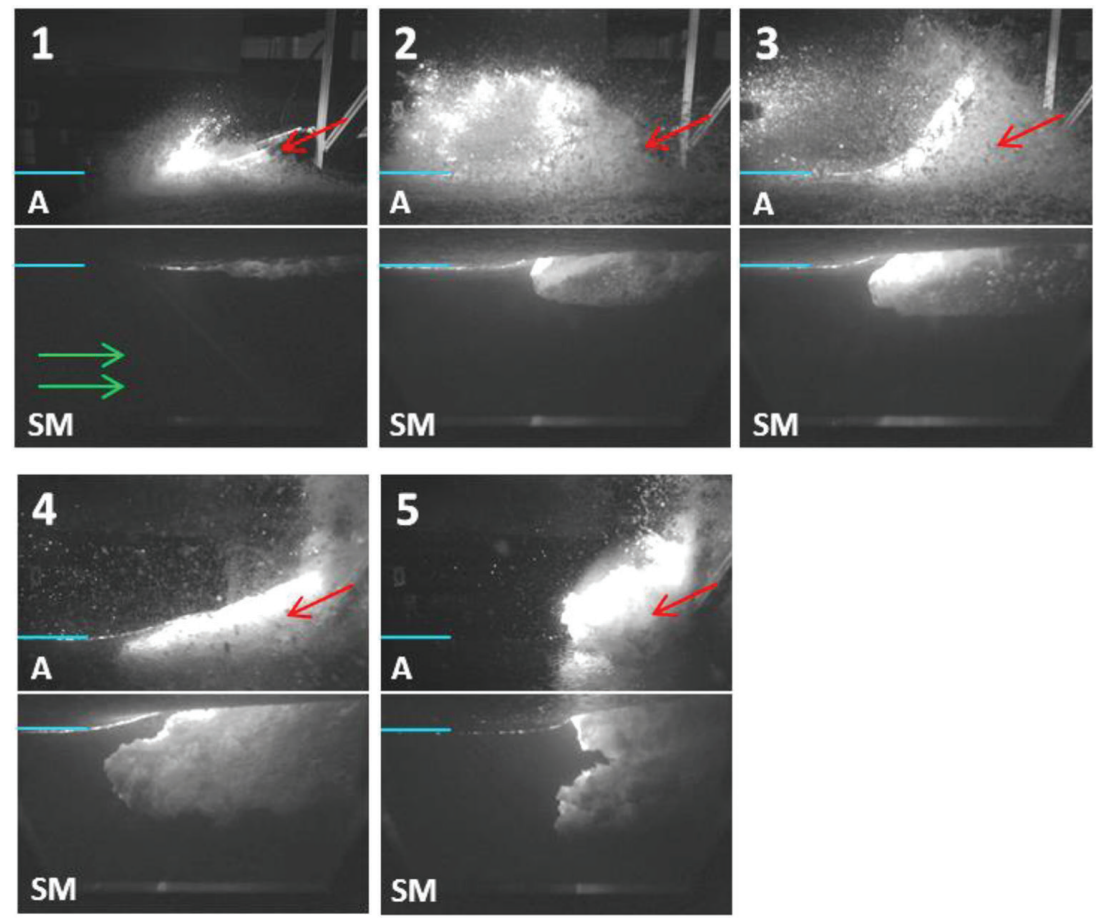

Figure 5: Life phases of the resurgent jets: impinging jet direction in red, free surface level in blue and counter-flow direction in green (A: aerial field, SM: submarine field). 
In blocked jet configuration, phenomenology is quite similar to the free jet condition. The difference relies on the perturbation induced by the plane shield located above the impact zone. Pressure signals (as shown in Fig. 6) highlight a transient phase with high levels due to the resurgent jet impact (dynamic peak) and a steady phase with lower levels. The steady phase features the same resurgent jet generation/destruction process (compared to the free jet conditions). Nevertheless, the deviation of the resurgent jet by the shield fastens the destruction phenomenon compared to the free jet configuration. As shown in Fig. 7 where the pressure field is interpolated between the sensors set, the impact zone due to the resurgent jet on the shield moves forward as the stagnation point penetrates the free surface.
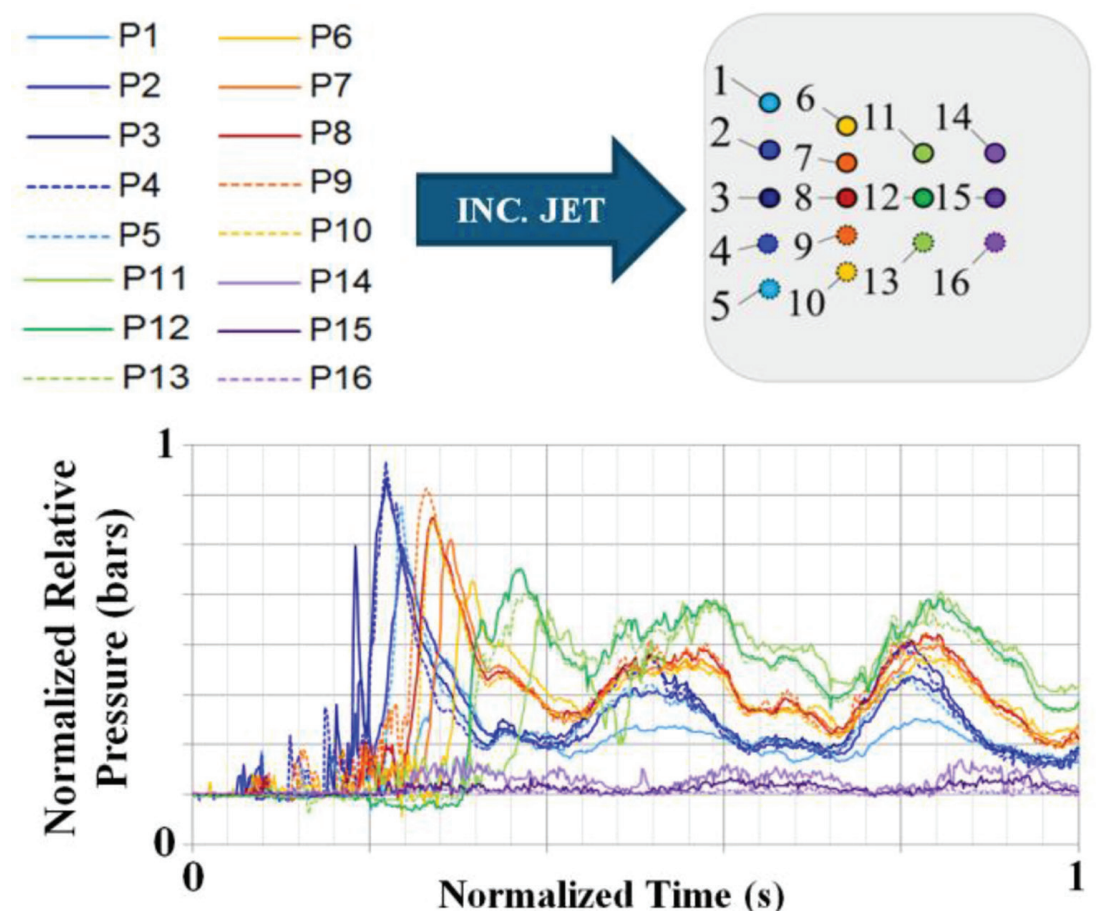

Figure 6: Pressure signals on the instrumented shield.


Figure 7: Wall pressure interpolated on the instrumented shield at different times. 
The transient phase observed with the pressure signals does not lead to maximum loads because of the low mass flow impinging the shield. Loads increase over time since the mass flow increases too. If the shield is closed enough to the free surface, a part of the resurgent jet mass is confined and contributes to loads. Note that the effort levels never drop to zero even if the resurgent jet is annihilated. Consequently, the shield location (both distances with the free surface and the impinging zone) is a key parameter to study the resurgent jet generation. This parameter modifies the phenomenon frequency and the type of recorded signals, as shown in Fig. 8: punctual (only one impact is recorded), periodic (the resurgent jet sweeping on the shield is clearly visible) or continuous (the resurgent jet is contained by the shield which leads to permanent loads; the phenomenon periodicity is still observable). The maximum effort and pressure are often obtained during the first period. In specific cases (the continuous configurations), maximum load values can be seen after the first period.

The test campaign also aims to assess critical parameters, which generate powerful resurgent jets. The influence of the injection pipe angle and diameter, the impinging jet velocity and the counter-flow velocity is studied. It appears that increasing the diameter results in strengthening the resurgent jet. Indeed, a more important mass quantity deflects the counter-flow. Similarly, reducing the impact angle enhances the momentum component value in the counter-flow direction. Increasing flow velocities modifies the energy levels and leads to more powerful resurgent jets. Velocity drives the phenomenon at the first order: the competition between the impinging jet and the counter-flow affects significantly the resurgent jet intensity. Two aspects must be considered:

1. The resurgent jet is a layer deflected by the impinging jet; therefore, this layer velocity is close to the counter-flow one. The higher the counter flow velocity is, the stronger the resurgent jet is.

2. The stagnation point dynamics between the impinging jet and the counter-flow: when the stagnation point is close to the surface, the deflected water mass flow is low and does not lead to important loads. On the contrary, if the stagnation point penetrates deeply the free surface, the deflection is less efficient due to the gravity. As a conclusion of the previous explanation, a penetration depth interval can be defined for which the resurgent jet load on the shield can be considered as maximum.

The location choice of the instrumented shield is a critical parameter as the resurgent jet signal may not be fully captured. Moreover, the nearest from the free surface the shield is, the highest the resurgent loads are, as the deflected water is more confined.
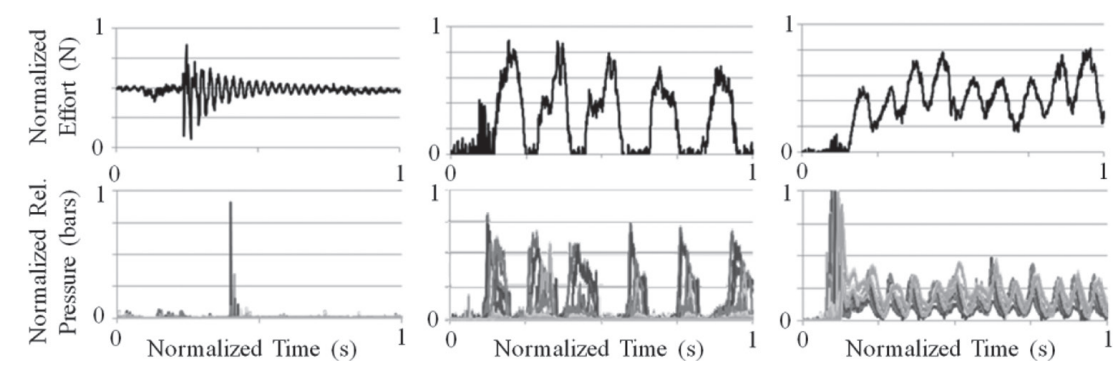

Figure 8: Kind of recorded signals (left: punctual, center: periodic, right: continuous). 


\section{NUMERICAL MODELS}

The simulations are computed with the code STARCCM+C). This multi-physics platform is chosen for its fluid models (gas or liquids, compressible or incompressible), its Eulerian multiphase approach and its VOF (Volume-Of-Fluid, [6]) technique dedicated to model the two-phase flow interface dynamics. The algorithmic structure based on a MPI protocol allows high parallelized computations, over 200 cores with an interesting scalability. Air is considered as an ideal gas and water as an incompressible medium. The interface between these two immiscible media is captured with a second order transport scheme, called HRIC (High Resolution Interface Capturing) respecting the VOF method formulation. The time integration scheme is implicit. A second order scheme is chosen with a controlled number of inner iterations ensuring the solution convergence for every single time step. Regarding the meshing approach, STARCCM+C is particularly efficient on polyhedral meshes (elements with 12 or 14 faces). These unstructured meshes seem to be more appropriate for complex flows with no specific flow directions. Plus, the numerical dissipation is limited with the dedicated numerical scheme implemented in STAR-CCM.

A pressure outlet condition is applied for the domain boundary conditions, except the bottom modelled by a wall condition. An injection pipe is located in the middle of the domain to generate the impinging jet (velocity and pressure are imposed). Depending on the studied configuration (free or blocked jet), an instrumented shield is added above the free surface (see Fig. 9). A size box, which allows a local mesh refinement, covers the injection pipe and the instrumented shield areas to capture the phenomenon. Two millions of cells compose the final mesh. In that numerical configuration, one second of physical time is computed in 12 hours with 256 cores.

\section{RESULTS AND DISCUSSIONS}

In this section, numerical results are discussed regarded to experiments. Calculations focused on the most dimensioning conditions of the test matrix. High levels of loads and pressures on the instrumented shield are recorded, as shown in Fig. 10. Simulations respect experimental conditions (distances, velocity inlet levels...). The free jet configuration (without instrumented shield) can be directly compared with video acquisition. Figure 11 illustrates the 3D free surface deformation, called resurgent jet.

Using a vertical plane cut, pressure field in the simulation with 50\%-VOF lines (which materialize the interface air-water) are shown over time in Fig. 12. Life phases of resurgent jet are similar to those identified with the experimental setup: the impact, the resurgent jet growth, the wave breaking, the interaction with impinging jet and finally the process restart.
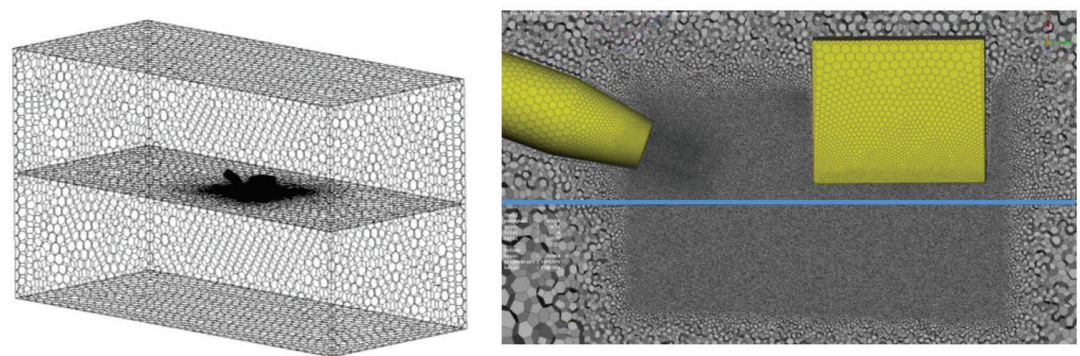

Figure 9: Polyhedral meshed domain (left) and zone of interest: injection pipe and instrumented shield, free surface on blue (right). 

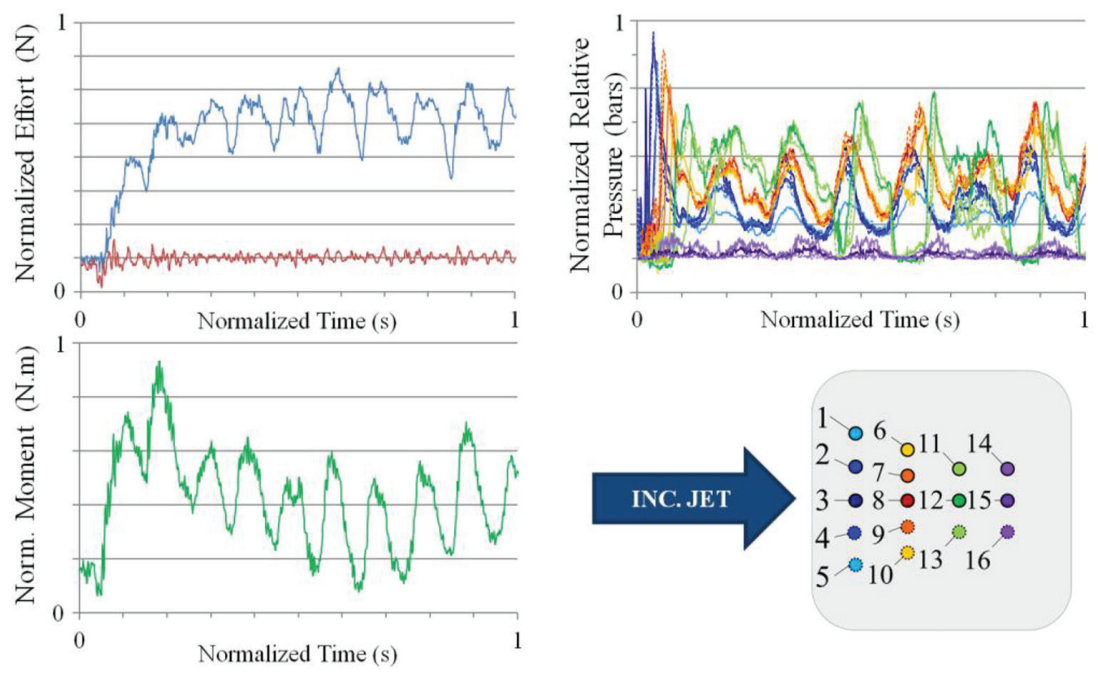

Figure 10: Recorded load and pressure signals.
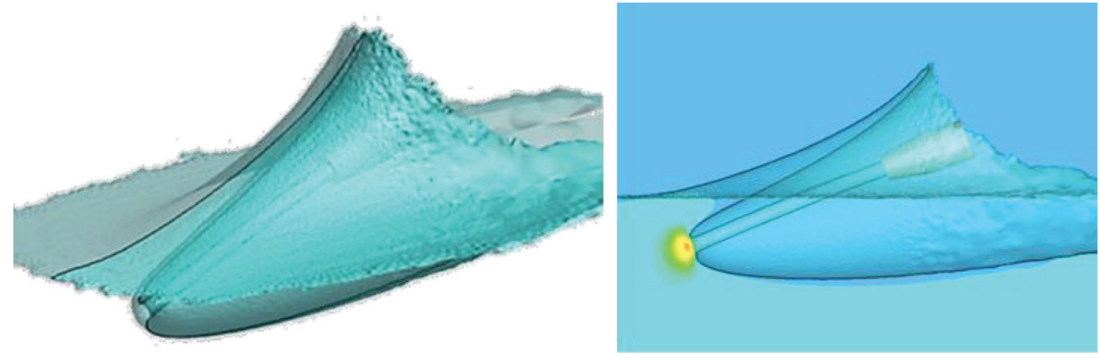

Figure 11: 3D deformation of the free surface - Resurgent jet.
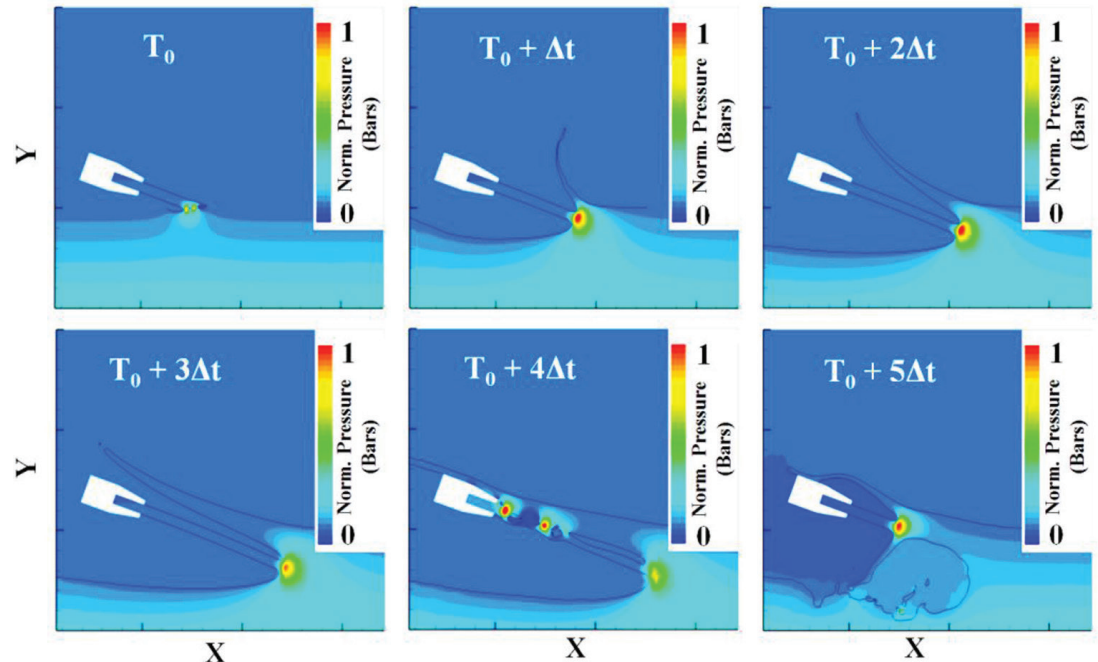

Figure 12: Pressure field in a vertical cut plane at different times. 
In order to extract the air-water interface, an image processing technique is applied on time resolved images acquisition. For some specific time step, this experimental interface is superposed on the 50\%-VOF isolines, as shown in Fig. 13. The computed resurgent jet has a velocity and dimensions close to experiment. Regarding to this figure, numerical and experimental results show a very good agreement in time and space as regards the free surface deformation complexity.

In addition to the free surface tracking, pressure and loads measurements extracted from the experimental test of a blocked jet configuration (i.e. equipped with the instrumented shield) can be compared with numerical simulation. Figure 14 depicts a 3D-illustration of the pressure field on the shield with the $50 \%$-VoF iso-surface at different times. The resurgent jet behavior is described in five mains steps. The jet is impinging the free surface (1) and generates the resurgent jet which impacts the instrumented shield (2). An overpressure front is

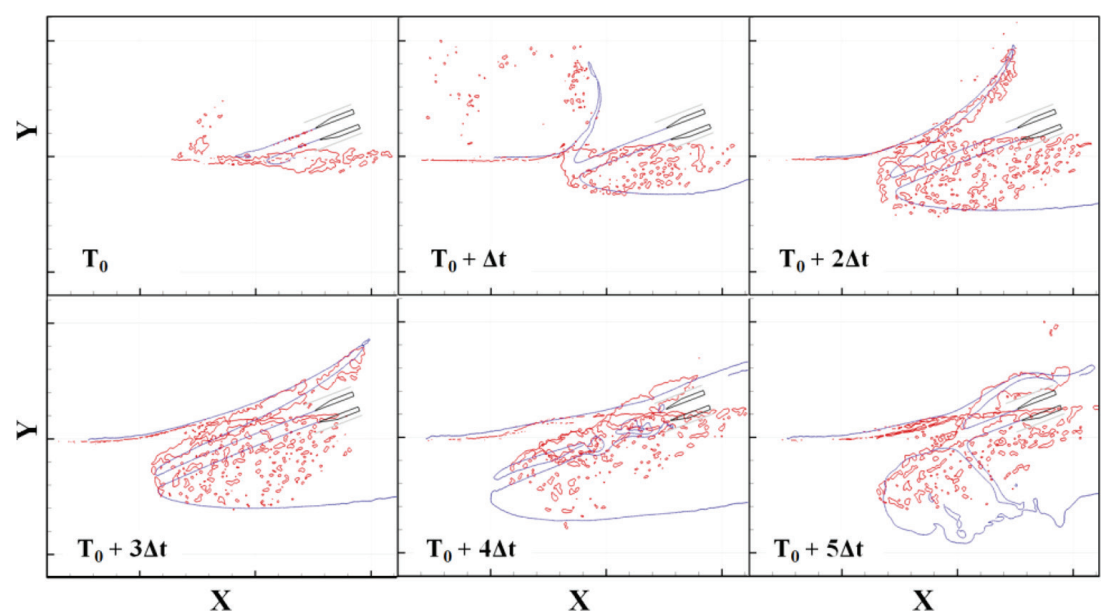

Figure 13: Comparison of the interface tracking (red: experiment with image processing, blue: simulation with VOF 50\% lines).
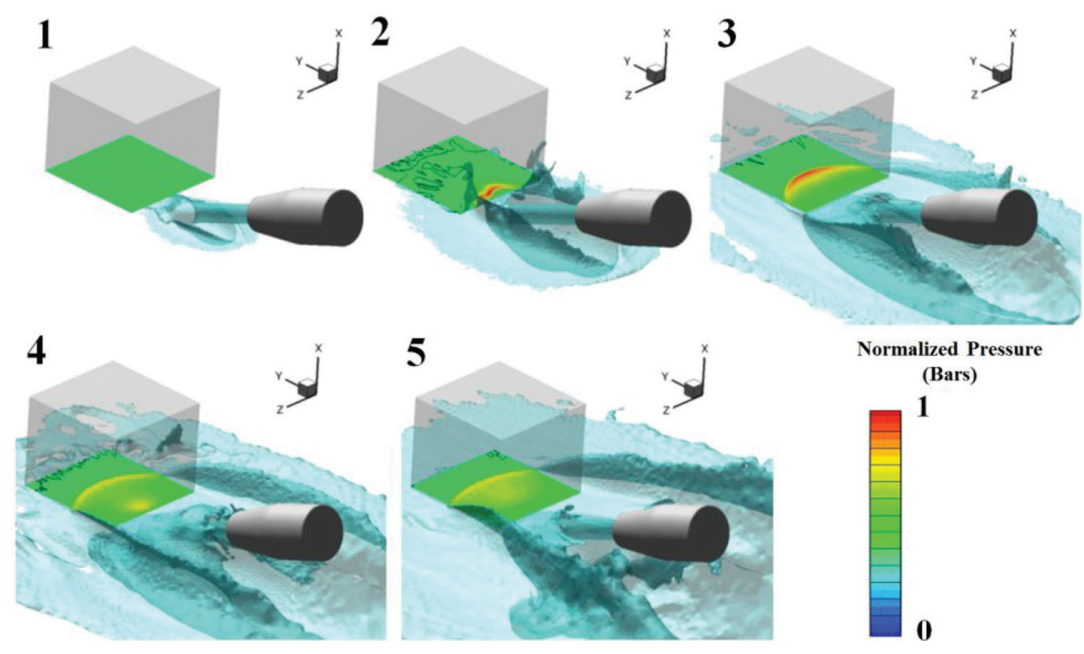

Figure 14: Pressure on instrumented shield and VOF-50\% iso-surface (blocked jet). 
moving forward (3) due the movement of the stagnation point (penetration of the impinging jet). The water deflected by the shield interacts with the impinging jet, then the resurgent jet losses intensity. The periodicity of the phenomenon generates another overpressure behind the first one (4) and moves towards it (5). The containment of water is observable and maintains an overpressure zone even when the resurgent jet is annihilated between each cycle.

A CFD/experiment comparison is then processed on the 2D pressure field measurements on the instrumented shield. Based on the 16 pressure probes measurements, a $2 \mathrm{D}$ field is interpolated and compared to the CFD pressure field. Results are presented for different instants in Fig. 15. The circular overpressure zone and its motion are clearly visible on the

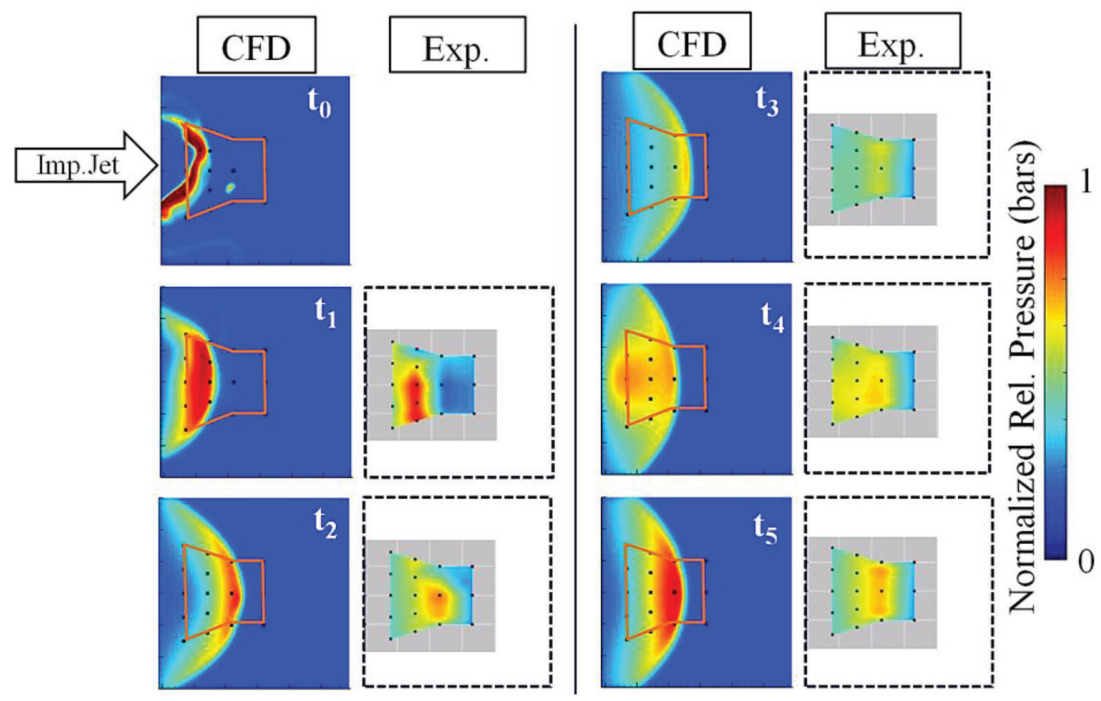

Figure 15: Pressure on instrumented shield (CFD and experiments).
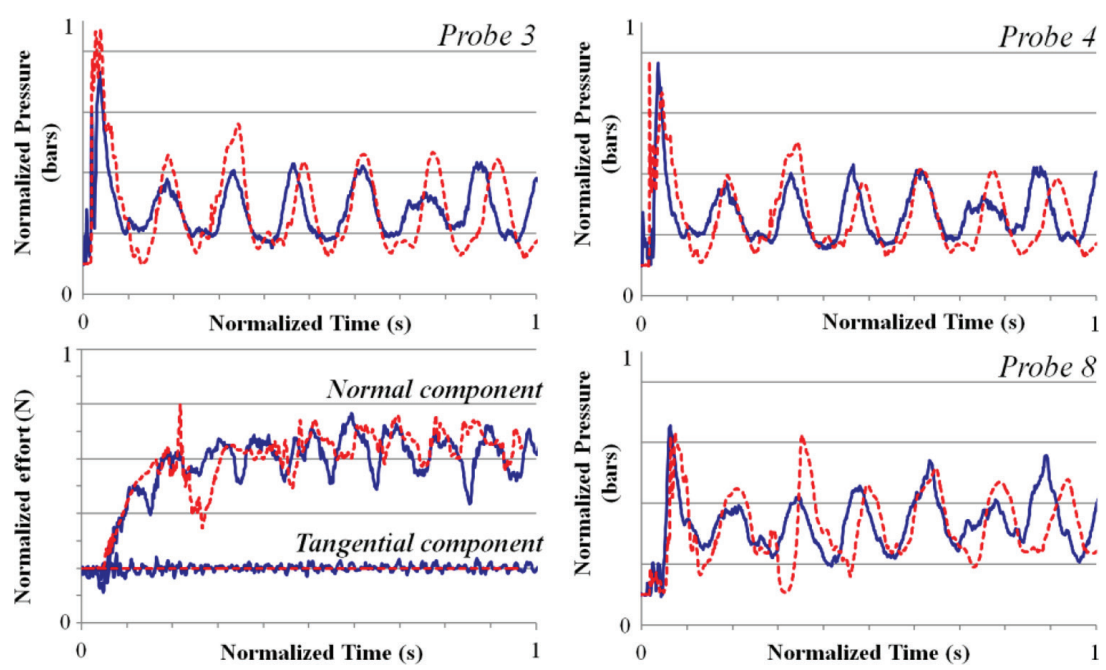

Figure 16: Pressure signals (top and bottom right corner) and resulting effort (bottom left corner) compared (blue: experiment, red dashed lines: simulation). 
simulation. The motion of this zone is also visible on the experiment and comparable to the simulation but its shape is not accurate due to the limited number of sensors.

Finally, experimental and numerical signals can be compared to assess the validity of the models used. As plotted on Fig. 16 for three different locations (probes 3, 4 and 8 represented in Fig. 10), pressure levels show a very good agreement in term of level and periodicity over time. Moreover, both tangential and normal component of the resulting efforts are in accordance between experiments and numerical approach.

\section{CONCLUSIONS}

The test campaign was a success since the phenomenon was clearly identified and showed unneglectable levels of resulting efforts and pressures. The conditions generating powerful resurgent jets are given by a high velocity of the reverse flow. Increasing diameter, velocity of the impinging jet and decreasing angle of impact are also dimensioning criteria. Considering situations where resurgent jets could occur, it must be taken into account for the design of industrial devices or off shore structures subject to breaking waves for example.

Both robustness and accuracy of the CFD approach agrees with the industrial needs to study complex configurations. The numerical approach matches very well compared to the experiments, as regards the interface tracking accuracy. Moreover, the level and the period of the resurgence generation are well captured. As a crucial industrial requirement, the efficient code scalability enables to reduce significantly the computation time.

The dual numerical and experimental approach highlights the influence of the impacting parameters. As a perspective, it will be interesting to test the efficiency of this method for coupled phenomena such as the oceanic swell disturbance on the resurgence generation.

\section{REFERENCES}

[1] McKeogh, E.J. \& Ervine, D.A., Air entrainment rate and diffusion pattern of plunging liquid jets. Chemical Engineering Science, 36(7), pp. 1161-1172, 1981.

https://doi.org/10.1016/0009-2509(81)85064-6

[2] Sene, K.J., Air entrainment by plunging jets. Chemical Engineering Science, 43(10), pp. 2615-2623, 1988.

https://doi.org/10.1016/0009-2509(88)80005-8

[3] Fontaine, E., Landrini, M. \& Tulin, M., On modeling the post breaking phase: splashing. Proceedings of 15th Workshop on Water Waves and Floating Bodies. Dan Caesarea, Israel, pp. 51-54, 2000.

[4] Deshpande, S.S., Trujillo, M.F., Wu, X. \& Chahine, G., Computational and experimental characterization of a liquid jet plunging into a quiescent pool at shallow inclination. International Journal of Heat and Fluid Flow, 34, pp. 1-14, 2012.

https://doi.org/10.1016/j.ijheatfluidflow.2012.01.011

[5] Gomez-Ledesma, R., An experimental investigation on the air entrainment by plunging jets, $\mathrm{PhD}$ thesis, Maryland University, 2004.

[6] Hirt, C.W. \& Nichols, B.D., Volume of fluid (VOF) method for the dynamics of free boundaries. Journal of Computational Physics, 39(1), pp. 201-225, 1981.

https://doi.org/10.1016/0021-9991(81)90145-5 